\title{
Derivative expansion and large gauge invariance at finite temperature
}

\author{
J. Barcelos-Neto* \\ Instituto de Física \\ Universidade Federal do Rio de Janeiro \\ RJ 21945-970 - Caixa Postal 68528 - Brasil \\ Ashok Das \\ Department of Physics and Astronomy \\ University of Rochester \\ Rochester, NY 14627 - USA
}

\begin{abstract}
We study the $0+1$ dimensional Chern-Simons theory at finite temperature within the framework of derivative expansion. We obtain various interesting relations, solve the theory within this framework and argue that the derivative expansion is not a suitable formalism for a study of the question of large gauge invariance.
\end{abstract}

PACS: 11.10.Ef, 11.10.Wx, 11.15.-q

*e-mail: barcelos@if.ufrj.br 


\section{Introduction}

The question of large gauge invariance in $2+1$ dimensional Chern-Simons theories at finite temperature has been of interest for some time now [1]-[6]. Simply put, at finite temperature, the coefficient of the Chern-Simons term becomes temperature dependent 4, 5] making it incompatible with the quantization condition necessary for large gauge invariance to hold. More recently, this question has been successfully analyzed [7] in the context of the $0+1$ dimensional Chern-Simons theory which suggests a possible solution for the puzzle of large gauge invariance in $2+1$ dimensions. Of particular interest is the fact that, at finite temperature, new terms seem to be generated in the effective action which are higher order (in the field variables) and are nonextensive in nature restoring the large gauge invariance in the full effective action. This is really a very interesting scenario and, consequently, this model has already been analyzed from other points of view [8] for a better understanding of various features and, furthermore, the results of this analysis have already been generalized to higher dimensions as well under various assumptions [9]-[12]. However, a complete and satisfactory understanding of the question of large gauge invariance in the $2+1$ dimensional theory is yet to come. And, for this reason, it is worth analyzing the new features in the $0+1$ dimensional model from as many points of view as is possible.

In this paper, we study this model within the framework of the derivative expansion [13, 14]. This is important from various points of view. First, one of the earliest calculations of the temperature dependence of the Chern-Simons coefficient was, in fact, in the context of the derivative expansion [5]. Second, in any higher dimensional theory, an exact solution is not possible. Consequently, one has to resort to some approximation scheme and the derivative expansion has proved to be an excellent approximation scheme in the study of several interesting phenomena. However, by construction, the effective action in the context of derivative expansion is an extensive quantity. And, so, the natural question that arises is how one would see nonextensive contributions to the effective action at finite temperature within this framework. Third, the $0+1$ dimensional model is free from the problems of nonanalyticity that plague the derivative expansion in higher dimensions at finite temperature [15]. In this paper, we solve the $0+1$ dimensional model at finite temperature within the framework of the derivative expansion. In some sense, this analysis is complementary to the one in ref. [8]. We derive various interesting relations that will be useful in the study of finite temperature field theory. Even though the $0+1$ dimensional model can be solved within this framework, we argue that the derivative expansion is not the appropriate framework for the study of large gauge invariance in higher dimensions. 


\section{Derivative expansion at $\mathrm{T}=0$}

The $0+1$ dimensional theory is described by the Lagrangian

$$
L=\bar{\psi}_{j}\left(i \partial_{t}-m-A\right) \psi_{j}-\kappa A
$$

where the flavor index, $j=1,2, \ldots, N_{f}$ is being summed. We follow the notations

of ref. [8] to which we refer the readers for details. The contribution of the fermions to the effective action is given by

$$
\begin{aligned}
\Gamma[A] & =-i \ln \left[\frac{\operatorname{det}\left(i \partial_{t}-m-A\right)}{\operatorname{det}\left(i \partial_{t}-m\right)}\right]^{N_{f}} \\
& =-i N_{f} \ln \operatorname{det}\left[1+i S_{F} A\right]
\end{aligned}
$$

which is normalized to vanish when the external gauge field, $A$, vanishes. Here, $S_{F}$ represents the fermion propagator.

Using the relation

$$
\operatorname{det} O=\exp \operatorname{Tr} \ln O
$$

we can write

$$
\begin{aligned}
\Gamma[A] & =-i N_{f} \operatorname{Tr} \ln \left[1+i S_{F} A\right] \\
& =-i N_{f} \operatorname{Tr}\left[i S_{F} A-\frac{1}{2} i S_{F} A i S_{F} A+\ldots\right]
\end{aligned}
$$

Here "Tr" simply stands for the trace in any complete basis (there is no Dirac index in $0+1$ dimensions). In the derivative expansion, $S_{F}(p)$ and $A$ are treated as noncommuting operators in (2.4) which can be commuted past each other through the use of the standard and canonical commutation relation

$$
A p=p A+i\left(\partial_{t} A\right)
$$

At zero temperature, the fermion propagator, in this model, is given by

$$
S_{F}(p)=\frac{i}{p-m+i \epsilon}
$$

so that the effective action at zero temperature (due to the fermions) has the form

$$
\Gamma[A]=-i N_{f} \operatorname{Tr}\left[-\frac{1}{p-m+i \epsilon} A-\frac{1}{2} \frac{1}{p-m+i \epsilon} A \frac{1}{p-m+i \epsilon} A+\cdots\right]
$$


If we look at the first term on the right hand side, we obtain

$$
\begin{aligned}
i N_{f} \operatorname{Tr} \frac{1}{p-m+i \epsilon} A & =i N_{f} \int \frac{d p}{2 \pi} \frac{1}{p-m+i \epsilon} \int d t A(t) \\
& =\frac{N_{f}}{2} \int d t A(t) \\
& =\frac{N_{f}}{2} a
\end{aligned}
$$

It is worth noting here that the integrand of the momentum integral does not satisfy the Cauchy convergence condition which implies that the correct value of the integral is obtained only after taking the contribution of the semi-circle into account.

The higher order terms in Eq. (2.7) can be brought to a form, through the use of Eq. (2.5), where all the momentum factors are at the left and, in general, will have the form

$$
N_{f} \operatorname{Tr} \sum_{n=2}^{\infty} \frac{1}{(p-m+i \epsilon)^{n}} f_{n}(A)=N_{f} \sum_{n=2}^{\infty} \int \frac{d p}{2 \pi} \frac{1}{(p-m+i \epsilon)^{n}} \int d t f_{n}(A)
$$

where $f_{n}(A)$ is a functional of $A$ involving derivatives as well. It is clear from the structure of the momentum integral in Eq. (2.9) that it vanishes. This is easily seen by closing the contour in the upper half of the complex plane in which case, there is no singularity inside the contour. Alternately,

$$
\begin{aligned}
\int \frac{d p}{2 \pi} \frac{1}{(p-m+i \epsilon)^{n}} & =\frac{1}{(n-1) !} \frac{\partial^{n-1}}{\partial m^{n-1}} \int \frac{d p}{2 \pi} \frac{1}{(p-m+i \epsilon)} \\
& =\frac{1}{(n-1) !} \frac{\partial^{n-1}}{\partial m^{n-1}}\left(-\frac{i}{2}\right)=0 \text { for } n \geq 2
\end{aligned}
$$

This shows that all the higher order terms in Eq. (2.7) which can be written in the form (2.9) vanish. Consequently, the complete contribution of the fermions to the effective action at zero temperature is given by

$$
\Gamma[A]=\frac{N_{f}}{2} \int d t A(t)=\frac{N_{f}}{2} a
$$

This is indeed the correct result. 


\section{Various relations}

At finite temperature, the fermion propagator is given in the real time formalism [16] by

$$
S_{F}(p)=\frac{i}{p-m+i \epsilon}-2 \pi n_{F}(m) \delta(p-m)
$$

where

$$
n_{F}(m)=\frac{1}{e^{\beta m}+1}
$$

is the Fermi statistical factor.

The derivative expansion, as we have seen, involves a product of propagators and, consequently, at finite temperature it would involve evaluating the integral of the product of a number of zero temperature propagators and a number of delta functions. These are extremely singular integrals and so, we need to define them carefully. In this section, we do precisely this which would help us in the analysis of the derivative expansion at finite temperature.

Let us denote a fermion loop (single flavor) with $N+1$ external photon lines with all possible insertions of the photon momenta by

$$
\begin{array}{r}
i \tilde{I}_{N+1}=-\frac{(-i)^{N+1}}{(N+1) !} \int \frac{d p}{2 \pi} \sum_{p e r m} S_{F}(p) S_{F}\left(p+k_{1}\right) S_{F}\left(p+k_{1}+k_{2}\right) \\
\cdots S_{F}\left(p+k_{1}+\cdots+k_{N}\right)
\end{array}
$$

Here "perm" stands for all possible permutations of the photon lines. The $N=0$ term, or the one point function is the simplest and has the form

$$
\begin{aligned}
i \tilde{I}_{1} & =i \int \frac{d p}{2 \pi}\left(\frac{i}{p-m+i \epsilon}-2 \pi n_{F}(m) \delta(p-m)\right) \\
& =\frac{i}{2}\left(1-2 n_{F}(m)\right)
\end{aligned}
$$

Any higher point function, on the other hand, will be a polynomial in $n_{F}(m)$ starting with the linear term (no constants). Each of these can be individually examined and since the power of $n_{F}(m)$ corresponds to the number of delta functions, we would have the necessary relations.

It is now straightforward to explicitly obtain the linear term in the $N+1 \mathrm{pt}$. function at lower orders. 


$$
\begin{aligned}
& i \tilde{I}_{2}^{(1)}=-\frac{i}{2 !}\left(\frac{1}{k+i \epsilon}-\frac{1}{k-i \epsilon}\right) n_{F}(m) \\
&=-\frac{i}{2 !}(-2 i \pi \delta(k)) n_{F}(m) \\
&=-\frac{1}{2} n_{F}(m)(2 \pi \delta(k)) \\
& i \tilde{I}_{3}^{(1)}=-\frac{i}{3 !}\left(\frac{1}{k_{1}+i \epsilon} \frac{1}{k_{2}+i \epsilon}-\frac{1}{k_{1}+i \epsilon} \frac{1}{k_{2}-i \epsilon}\right. \\
&\left.-\frac{1}{k_{1}-i \epsilon} \frac{1}{k_{2}+i \epsilon}+\frac{1}{k_{1}-i \epsilon} \frac{1}{k_{2}-i \epsilon}\right) n_{F}(m) \\
& i \tilde{I}_{4}^{(1)}=-\frac{1}{4 !} \frac{1}{\left(k_{1}+i \epsilon\right)\left(k_{2}+i \epsilon\right)\left(k_{3}+i \epsilon\right)} \\
&-\frac{1}{k_{1}-i \epsilon} \frac{1}{\left(k_{2}+i \epsilon\right)\left(k_{3}+i \epsilon\right)} \\
&-\frac{1}{k_{2}-i \epsilon} \frac{1}{\left(k_{3}+i \epsilon\right)\left(k_{1}+i \epsilon\right)} \\
&-\frac{1}{k_{3}-i \epsilon} \frac{1}{\left(k_{1}+i \epsilon\right)\left(k_{2}+i \epsilon\right)} \\
&+\frac{1}{\left(k_{1}-i \epsilon\right)\left(k_{2}-i \epsilon\right)} \frac{1}{k_{3}+i \epsilon} \\
&+\frac{1}{\left(k_{3}-i \epsilon\right)\left(k_{1}-i \epsilon\right)} \frac{1}{k_{2}+i \epsilon} \\
&+\frac{1}{\left(k_{2}-i \epsilon\right)\left(k_{3}-i \epsilon\right)} \frac{1}{k_{1}+i \epsilon} \\
&\left.-\frac{1}{\left(k_{1}-i \epsilon\right)\left(k_{2}-i \epsilon\right)\left(k_{3}-i \epsilon\right)}\right] n_{F}(m) \\
&= \\
&= \\
&= \\
&= \\
&=
\end{aligned}
$$

and so on. The general pattern of terms for the linear term is now clear. Let

$$
J_{n}\left(k_{1}, k_{2}, \ldots, k_{n}\right)=\frac{1}{\left(k_{1}+i \epsilon\right)\left(k_{2}+i \epsilon\right) \cdots\left(k_{n}+i \epsilon\right)}
$$

Then, we can write

$$
\begin{aligned}
i \tilde{I}_{N+1}^{(1)}= & -\frac{i}{(N+1) !}\left[J_{N}\left(k_{1}, k_{2}, \ldots, k_{N}\right)\right. \\
& -\frac{1}{k_{1}-i \epsilon} J_{N-1}\left(k_{2}, k_{3}, \ldots, k_{N}\right)-\cdots-\frac{1}{k_{N}-i \epsilon} J_{N-1}\left(k_{1}, k_{2}, \ldots, k_{N-1}\right) \\
& +\frac{1}{\left(k_{1}-i \epsilon\right)\left(k_{2}-i \epsilon\right)} J_{N-2}\left(k_{3}, \ldots, k_{N}\right)+\cdots \\
& +\frac{1}{\left(k_{N-1}-i \epsilon\right)\left(k_{N}-i \epsilon\right)} J_{N-2}\left(k_{1}, \ldots, k_{N-2}\right)-\cdots \\
& +\cdots
\end{aligned}
$$




$$
\left.+(-1)^{N} \frac{1}{\left(k_{1}-i \epsilon\right)\left(k_{2}-i \epsilon\right) \cdots\left(k_{N}-i \epsilon\right)}\right] n_{F}(m)
$$

From the definition in (3.6), we see that we can combine terms pairwise in (3.7) to write

$$
\begin{aligned}
i \tilde{I}_{N+1}^{(1)}= & -\frac{i}{(N+1) !}\left(\frac{1}{k_{1}+i \epsilon}-\frac{1}{k_{1}-i \epsilon}\right)\left[J_{N-1}\left(k_{2}, k_{3}, \ldots, k_{N}\right)\right. \\
& -\frac{1}{k_{2}-i \epsilon} J_{N-2}\left(k_{3}, \ldots, k_{N}\right)-\cdots-\frac{1}{k_{N}-i \epsilon} J_{N-1}\left(k_{2}, \ldots, k_{N-1}\right) \\
& +\cdots \\
& \left.+(-1)^{N-1} \frac{1}{\left(k_{2}-i \epsilon\right) \cdots\left(k_{N}-i \epsilon\right)}\right] n_{F}(m) \\
= & \frac{1}{N+1}\left(-2 i \pi \delta\left(k_{1}\right)\right) i \tilde{I}_{N}^{(1)}
\end{aligned}
$$

Upon iteration, this gives

$$
\begin{aligned}
i \tilde{I}_{N+1}^{(1)} & =\frac{(-i)^{N+1}}{(N+1) !}\left(2 \pi \delta\left(k_{1}\right)\right)\left(2 \pi \delta\left(k_{2}\right)\right) \cdots\left(2 \pi \delta\left(k_{N}\right)\right) n_{F}(m) \\
& =i I_{N+1}^{(1)}\left(2 \pi \delta\left(k_{1}\right)\right) \cdots\left(2 \pi \delta\left(k_{N}\right)\right)
\end{aligned}
$$

We can, similarly, analyze the terms quadratic and cubic in $n_{F}(m)$. The details are not very illuminating and so we only give the final results.

$$
\begin{aligned}
& i \tilde{I}_{N+1}^{(2)}=i I_{N+1}^{(2)}\left(2 \pi \delta\left(k_{1}\right)\right) \cdots\left(2 \pi \delta\left(k_{N}\right)\right) \\
& i \tilde{I}_{N+1}^{(3)}=i I_{N+1}^{(3)}\left(2 \pi \delta\left(k_{1}\right)\right) \cdots\left(2 \pi \delta\left(k_{N}\right)\right)
\end{aligned}
$$

where

$$
\begin{aligned}
i I_{N+1}^{(2)}= & -\frac{(-i)^{N+1}}{(N+1) !}\left({ }^{N} C_{1}+{ }^{N} C_{2}+\cdots+{ }^{N} C_{N}\right)\left(n_{F}(m)\right)^{2} \\
= & -\frac{(-i)^{N+1}}{(N+1) !}\left(2^{N}-1\right)\left(n_{F}(m)\right)^{2} \\
i I_{N+1}^{(3)}= & \frac{(-i)^{N+1}}{(N+1) !}\left[{ }^{N} C_{1}\left({ }^{N-1} C_{1}+{ }^{N-1} C_{2}+{ }^{N-1} C_{3}+\cdots+{ }^{N-1} C_{N-1}\right)\right. \\
& +{ }^{N} C_{2}\left({ }^{N-2} C_{1}+{ }^{N-2} C_{2}+{ }^{N-2} C_{3}+\cdots+{ }^{N-2} C_{N-2}\right)
\end{aligned}
$$




$$
\begin{gathered}
+\cdots \\
\left.+{ }^{N} C_{N-1}\left({ }^{1} C_{1}\right)\right]\left(n_{F}(m)\right)^{3} \\
=\frac{(-i)^{N+1}}{(N+1) !}\left(3^{N}-2 \cdot 2^{N}+1\right)\left(n_{F}(m)\right)^{3}
\end{gathered}
$$

In this way, we can derive the general formula that (for $r \leq N+1$ and $r \geq 1$ )

$$
i \tilde{I}_{N+1}^{(r)}=i I_{N+1}^{(r)}\left(2 \pi \delta\left(k_{1}\right)\right) \cdots\left(2 \pi \delta\left(k_{N}\right)\right)
$$

where

$$
\begin{aligned}
i I_{N+1}^{(r)} & =(-1)^{r+1} \frac{(-i)^{N+1}}{(N+1) !}\left(n_{F}(m)\right)^{r} \sum_{s=0}^{r-1}(-1)^{s-1} C_{s}(r-s)^{N} \\
& =(-1)^{r+1} \frac{(-i)^{N+1}}{(N+1) !}\left(n_{F}(m)\right)^{r} b_{N+1}^{(r)}
\end{aligned}
$$

so that the $N+1 \mathrm{pt}$. function can be written as $\left(\tilde{I}_{1}=I_{1}\right)$

$$
\begin{aligned}
i \tilde{I}_{N+1} & =\left(2 \pi \delta\left(k_{1}\right)\right) \cdots\left(2 \pi \delta\left(k_{N}\right)\right) \sum_{r=1}^{N+1} i I_{N+1}^{(r)} \\
& =\left(2 \pi \delta\left(k_{1}\right)\right) \cdots\left(2 \pi \delta\left(k_{N}\right)\right) i I_{N+1}
\end{aligned}
$$

The results in (3.12) $-(3.14)$ are interesting from various points of view. First, from the explicit form of the result in (3.14), we see that

$$
k_{i} \tilde{I}_{N+1}\left(k_{1}, \ldots, k_{N}\right)=0 \quad \text { for } i=1,2, \ldots, N
$$

verifying explicitly that the amplitudes satisfy the Ward identities of the theory. In this sense, it is complementary to the analysis in ref. [8] where the Ward identities were used to carry out the calculations. Second, from the properties of the binomial coefficients, it is straightforward to see that

$$
b_{N+1}^{(r)}=(r-1) b_{N}^{(r-1)}+r b_{N}^{(r)}
$$

which leads to the relation that

$$
\frac{\partial I_{N}}{\partial m}=-i \beta(N+1) I_{N+1}
$$


which is the recursion relation derived in ref. [8] following from the Ward identity and we can identify $\tilde{I}_{N}$ with the vertex functions obtained in ref. [8] up to a flavor factor. Finally, if we set all the external momenta to zero, then from (3.13), we obtain (for $r \leq N+1$ and $r \geq 1$ )

$$
\int \frac{d p}{2 \pi} \frac{1}{(p-m+i \epsilon)^{N+1-r}} \delta^{r}(p-m)=(-i)^{N+1-r} b_{N+1}^{(r)}(2 \pi \delta(0))^{N}
$$

This defines the singular integrals consistent with the Ward identity. With these relations, we are now ready to analyze the derivative expansion at finite temperature.

\section{Derivative expansion at $\mathbf{T} \neq 0$}

Going back to the definition of the derivative expansion in (2.4), we note that the first term in the expansion is again quite simple at finite temperature (as is derived in (3.4) also).

$$
\begin{aligned}
\Gamma_{1}[A] & =-i N_{f} \operatorname{Tr} i S_{F}(p) A(t) \\
& =-i N_{f} \int \frac{d p}{2 \pi}\left(-\frac{1}{p-m+i \epsilon}-2 i \pi n_{F}(m) \delta(p-m)\right) \int d t A(t) \\
& =\frac{N_{f}}{2}\left(1-2 n_{F}(m)\right) \int d t A(t) \\
& =\frac{N_{f}}{2}\left(1-2 n_{F}(m)\right) a \\
& =N_{f} I_{1} a
\end{aligned}
$$

It is at the second and higher orders that we run into the conceptual question of how a single time integral can become a nonextensive term. Therefore, let us analyze the second order term in some detail.

$$
\begin{aligned}
\Gamma_{2}[A] & =i \frac{N_{f}}{2} \operatorname{Tr} i S_{F}(p) A\left(i S_{F}(p)\right) A \\
& =-i \frac{N_{f}}{2} \operatorname{Tr} S_{F}(p) S_{F}\left(p+i \partial_{1}\right) A_{1} A_{2}
\end{aligned}
$$

Here we have used the commutation relations in (2.5) to move all the momentum dependent factors to the left and have labeled the fields $A$ merely to indicate that the derivative acts only on the first of the two fields. The trace can now be taken and the momentum integral can be done using (3.14) which gives 


$$
\Gamma_{2}[A]=N_{f} I_{2} \int d t 2 \pi\left(\delta\left(\partial_{t}\right) A\right) A
$$

This is a single integral, but the integrand is quite unusual. It is clear from the properties of delta functions that $\left(\delta\left(\partial_{t}\right) A\right)$ cannot depend on $t$. In fact, it is much simpler to analyze this in the Fourier transformed space where it becomes

$$
\begin{aligned}
\Gamma_{2}[A] & =N_{f} I_{2} \int \frac{d p}{2 \pi} 2 \pi \delta(k) \tilde{A}(k) \tilde{A}(-k) \\
& =N_{f} I_{2} \tilde{A}(0) \tilde{A}(0) \\
& =N_{f} I_{2}\left(\int d t A(t)\right)^{2} \\
& =N_{f} I_{2} a^{2}
\end{aligned}
$$

This is quite interesting and clarifies how a single time integral becomes a nonextensive term in the effective action. This also brings out another interesting point. Namely, had we expanded the second propagator in (4.2) in powers of the derivatives, as the philosophy of the derivative expansion would dictate, we would have obtained an infinite series of local terms. Each momentum integral, in this case, can be done using (3.17). If we sum this series, of course, we would obtain the nonextensive term in (4.4). However, if we were to truncate the powers of the derivative at some order, as would become the case in any realistic, nontrivial model, we would miss completely the nonextensive nature of the effective action. The nonextensive structure is, of course, quite crucial in restoring the large gauge invariance. So, it would appear that the derivative expansion is not an ideal setting to discuss the question of large gauge invariance.

To complete this calculation, then, at the $(N+1)$ th order, if we commute all the propagators to the left and do the momentum integral using (3.13), we would obtain

$$
\begin{aligned}
\Gamma_{N+1} & =N_{f} I_{N+1} \int d t\left(2 \pi \delta\left(\partial_{t}\right) A\right) \cdots\left(2 \pi \delta\left(\partial_{t}\right) A\right) A \\
& =N_{f} I_{N+1}\left(\int d t A(t)\right)^{N+1} \\
& =N_{f} I_{N+1} a^{N+1}
\end{aligned}
$$

(The correct combinatoric factor arises from appropriate symmetrization.) This is exactly the result obtained in ref. [8] (remember that $N_{f} I_{f} \rightarrow I_{N}$ of ref. [8]) and hence the series can again be summed to give

$$
\begin{aligned}
\Gamma[A] & =\sum_{N=1}^{\infty} \Gamma_{N} \\
& =-i N_{f} \ln \left(\cos \frac{a}{2}+i \tanh \frac{\beta m}{2} \sin \frac{a}{2}\right)
\end{aligned}
$$


which is the exact result [7].

\section{Conclusion}

In this paper, we have solved the $0+1$ dimensional Chern-Simons theory within the framework of the derivative expansion. We have derived various interesting relations that will be useful in the study of finite temperature field theory. We have also explicitly clarified how a single time integral gives rise to nonextensive terms in the effective action. It appears from this analysis that the derivative expansion is not an ideal setting to examine the question of large gauge invariance in higher dimensions.

Acknowledgment: A.D. would like to thank the members of the Department of Theoretical Physics in UFRJ for hospitality where this work was done. J.B.-N. is supported in part by Conselho Nacional de Desenvolvimento Científico e Tecnológico - CNPq, Financiadora de Estudos e Projetos - FINEP, and Fundação Universitária José Bonifácio - FUJB (Brazilian Research Agencies). A.D. is supported in part by US DOE Grant No. DE-FG-02-91ER40685, NSF-INT-9602559 and a Fulbright grant.

\section{References}

[1] S. Deser, R. Jackiw and S. Templeton, Phys. Rev. Lett. 48 (1982) 975; Ann. Phys. 140 (1982) 372.

[2] A. Redlich, Phys. Rev. Lett. 52 (1984) 18; Phys. Rev. D29 (1984) 2366.

[3] R.D. Pisarski, Phys. Rev. D35 (1987) 664.

[4] K.T. Ishikawa and A. Matsuyama, Nucl. Phys. B280 (1987) 523.

[5] K.S. Babu, A. Das and P. Panigrahi, Phys. Rev. D36 (1987) 3725.

[6] N. Bralić, C. Fosco and F. Schaposnik, Phys. Lett. B383 (1996) 199; D. Cabra, E. Fradkin, G. Rossini and F. Schaposnik, Phys. Lett. B383 (1996) 434.

[7] G. Dunne, K. Lee and C. Lu, Phys. Rev. Lett. 78 (1997) 3434.

[8] A. Das and G. Dunne, hep-th/9712144, to appear in Phys. Rev. D.

[9] S. Deser, L. Griguolo and D. Seminara, Phys. Rev. Lett. 79 (1997) 1976; S. Deser, L. Griguolo and D. Seminara, hep-th/9712066.

[10] C. Fosco, G. Rossini and F. Schaposnik, Phys. Rev. Lett. 79 (1997) 1980; Phys. Rev. D56 (1997) 6547. 
[11] I. Aitchison and C. Fosco, hep-th/9709035.

[12] R. Gonzáles-Felipe, hep-th/9709079.

[13] I.Aitchison and C. Fraser, Phys. Lett. 146B (1984) 63.

[14] A. Das and A. Karev, Phys. Rev. D36 (1987) 623; Phys. Rev. D36 (1987) 2591.

[15] A. Das and M. Hott, Phys. Rev. D50 (1994) 6655.

[16] A. Das, Finite temperature field theory, World Scientific (1997) 